\title{
The Relationship of Parathyroid Activity to Pyrophosphate Excretion*
}

\author{
Louis V. Avioli, $\dagger$ Joseph E. McDonald, Philip H. Henneman, \\ AND SOOK WON LEE \\ (From the New Jersey College of Medicine, Jersey City, N. J.)
}

Recent observations in this laboratory suggested that urinary pyrophosphate excretion may reflect the rate of bone resorption in clinical disorders of bone metabolism (1). Since parathyroid hormone is known to accelerate bone resorption $(2,3)$, changes in parathyroid function might also be expected to be associated with parallel changes in pyrophosphate excretion. An elevated urinary excretion of pyrophosphate has been previously reported in patients with hyperparathyroidism (1). The present studies were designed to further examine the relationship of parathyroid activity to pyrophosphate excretion.

\section{Methods}

Four different types of experiments were done:

1) Measurements of urinary pyrophosphate during periods of active bone resorption and formation. Urinary pyrophosphate, calcium, and hydroxyproline measurements were made in two patients with active Paget's disease (F.H. and D.F.) before, during, and after 12 days of relative immobilization induced by bed rest. Similar measurements were also made in two patients with hyperparathyroidism (H.G. and E.F.) before and during a 6-day interval immediately after remedial parathyroid surgery. All four subjects were studied during periods of hospitalization after 7 to 14 days adaptation to constant weighed diets containing $800 \mathrm{mg}$ of $P$ and $600 \mathrm{mg}$ of $\mathrm{Ca}$ and devoid of fish, jello, ice cream, or other gelatin-rich foods. Urine was collected in 24-hour pools, and serial blood specimens were obtained daily.

* Submitted for publication January 3, 1966; accepted March 12, 1966.

Supported in part by grants from the U. S. Public Health Service (Clinical Research Center grant FR-41, National Institute of Arthritis and Metabolic Diseases grant 06404, and training grant AM-5449) and by Atomic Energy Commission contract AT (30-1)-3174.

$\dagger$ Recipient of a Career Research Development Award of the National Institute of General Medical Sciences (6-K3-GM-22, 676-02).

Address requests for reprints to Dr. Louis V. Avioli, The Jewish Hospital, 216 S. Kingshighway. St. Louis, Mo. 63110.
2) Four-hour calcium infusion. Fifteen $\mathrm{mg}$ of calcium (as calcium glucoheptonate) per $\mathbf{k g}$ of body weight was infused intravenously to four patients with untreated idiopathic hypoparathyroidism (ages 21 to 37 years), two patients with hyperparathyroidism (ages 47 and 63 years), and four normal volunteers (ages 23 to 30 years). All subjects were maintained on constant dietary intakes containing 700 to $800 \mathrm{mg} \mathrm{P}, 500$ to $600 \mathrm{mg} \mathrm{Ca}$, and less than $10 \mathrm{mg}$ of hydroxyproline in the form of protein. On day 7 of the diet, the calcium was administered in $0.9 \%$ sodium chloride solution by constant infusion over a 4-hour period beginning at 8:00 a.m. Four-hour urine collections were initiated at 8:00 a.m. on the day before the calcium infusion and continued for the subsequent 72-hour period. Blood samples were obtained in the middle of each 4-hour collection period. Calcium glucoheptonate was also administered by continuous intravenous infusion to one patient (N.D.) with untreated idiopathic hypoparathyroidism for 4 consecutive days. The 5 days before and 5 days after the infusion served as control periods. The dosage of calcium averaged $16.2 \mathrm{mg}$ per $\mathrm{kg}$ per day. Urine was collected in 24-hour pools, and blood samples were obtained daily.

3) Parathyroid extract administration. Aqueous PTE 1 was administered to two normal male subjects and to four patients with untreated idiopathic hypoparathyroidism. PTE was given intramuscularly at a dosage of $200 \mathrm{U}$ every 8 hours for a period of 6 days. These six patients were also studied during constant dietary intakes identical to those reported above in the calcium infusion experiments. Urine samples were collected in 24-hour pools and blood samples obtained at daily intervals.

In each of the above experiments measurements of $\mathrm{Ca}$, $P$, and creatinine were made on blood and urine. In addition, pyrophosphate and total hydroxyproline measurements were made on all urine specimens.

4) Renal arterial perfusion with parathyroid extract. Five female mongrel dogs weighing 15 to $22 \mathrm{~kg}$ were anesthetized with 10 to $15 \mathrm{mg}$ per $\mathrm{kg}$ of pentobarbital intravenously. Both renal arteries and ureters were exposed via a subdiaphragmatic abdominal approach and the ureters catheterized, thus permitting simultaneous collection of timed urine samples from both kidneys. After identification and exposure of a renal artery, the blood flow was temporarily interrupted by an appropriately placed ligature. A special cannula consisting of an

1 Eli Lilly, Indianapolis, Ind. 
TABLE I

Urinary pyrophosphate, orthophosphate, hydroxyproline, and calcium in hyperparathyroidism after surgery and Paget's disease during immobilization*

\begin{tabular}{|c|c|c|c|c|c|c|c|}
\hline Patient & Diagnosis & Status & PPi & $\mathrm{OH}-\mathrm{Pr}$ & $\mathrm{Ca}$ & $\mathbf{P i}$ & $\mathrm{C}_{\mathrm{s}} \dagger$ \\
\hline H.G. & Hyperparathyroidism & $\begin{array}{l}\text { Preoperativeł } \\
\text { Postoperative§ }\end{array}$ & $\begin{array}{r}m g P / 24 \mathrm{hr} \\
25.7 \pm 1.2 \\
7.8 \pm 0.7\end{array}$ & $\begin{array}{l}m g / 24 \mathrm{hr} \\
89 \pm 5 \\
45 \pm 3\end{array}$ & $\begin{array}{c}m g / 24 h r \\
483 \pm 20 \\
61 \pm 9\end{array}$ & $\begin{array}{l}m g / 24 h r \\
894 \pm 64 \\
452 \pm 34\end{array}$ & $\begin{array}{c}m g / 100 m l \\
15.0-17.7(4) \\
8.2-10.9(7)\end{array}$ \\
\hline S.F. & Hyperparathyroidism & $\begin{array}{l}\text { Preoperative } \\
\text { Postoperative }\end{array}$ & $\begin{array}{r}12.3 \pm 1.8 \\
3.5 \pm 0.4\end{array}$ & $\begin{array}{r}128 \pm 6 \\
35 \pm 2\end{array}$ & $\begin{array}{l}58 \pm 4 \\
10 \pm 2\end{array}$ & $\begin{array}{r}1,330 \pm 52 \\
657 \pm 21\end{array}$ & $\begin{array}{r}10.3-10.6(5) \\
6.5-6.8(5)\end{array}$ \\
\hline D.F. & Paget's disease & $\begin{array}{l}\text { Control } \| \\
\text { Bed rest } \| \\
\text { Postcontrol } \|\end{array}$ & $\begin{array}{l}25.9 \pm 1.1 \\
48.1 \pm 6.1 \\
18.7 \pm 2.7\end{array}$ & $\begin{array}{l}175 \pm 4 \\
225 \pm 9 \\
156 \pm 5\end{array}$ & $\begin{array}{r}87 \pm 6 \\
117 \pm 4 \\
89 \pm 3\end{array}$ & $\begin{array}{l}692 \pm 43 \\
671 \pm 26 \\
702 \pm 12\end{array}$ & $\begin{array}{l}8.8-9.2(6) \\
9.2-9.5(6) \\
8.8-9.4(6)\end{array}$ \\
\hline F.H. & Paget's disease & $\begin{array}{l}\text { Control } \| \\
\text { Bed rest } \| \\
\text { Postcontrol } \|\end{array}$ & $\begin{array}{l}18.6 \pm 2.9 \\
28.0 \pm 1.7 \\
18.9 \pm 1.3\end{array}$ & $\begin{array}{l}256 \pm 8 \\
324 \pm 6 \\
248 \pm 12\end{array}$ & $\begin{array}{l}125 \pm 3 \\
159 \pm 7 \\
129 \pm 6\end{array}$ & $\begin{array}{l}669 \pm 14 \\
562 \pm 21 \\
639 \pm 15\end{array}$ & $\begin{array}{l}8.4-8.9(6) \\
8.8-9.2(8) \\
8.7-9.6(8)\end{array}$ \\
\hline
\end{tabular}

* Abbreviations: $\mathrm{PPi}=$ inorganic pyrophosphate; $\mathrm{Pi}=$ inorganic orthophosphate; $\mathrm{OH}-\mathrm{Pr}=$ total hydroxyproline $\mathrm{C}_{\mathrm{s}}=$ serum calcium; $\mathrm{Ca}=$ urine calcium.

$\dagger$ Range of serum calciums. Number of determinations noted in parentheses.

Urinary values represent the mean and standard error of 3 consecutive 24-hour collections.

$\S$ Urinary values represent the mean and standard error of 6 consecutive 24 -hour collections.

I| Urinary values represent the mean and standard error of 12 consecutive 24-hour collections.

outer cannula of 18-gauge polyvinyl tubing and an inner metal needle was then inserted into the renal artery by a modification of the technique of Pullman, Lavender, Aho, and Rasmussen (4). The inner needle was with-
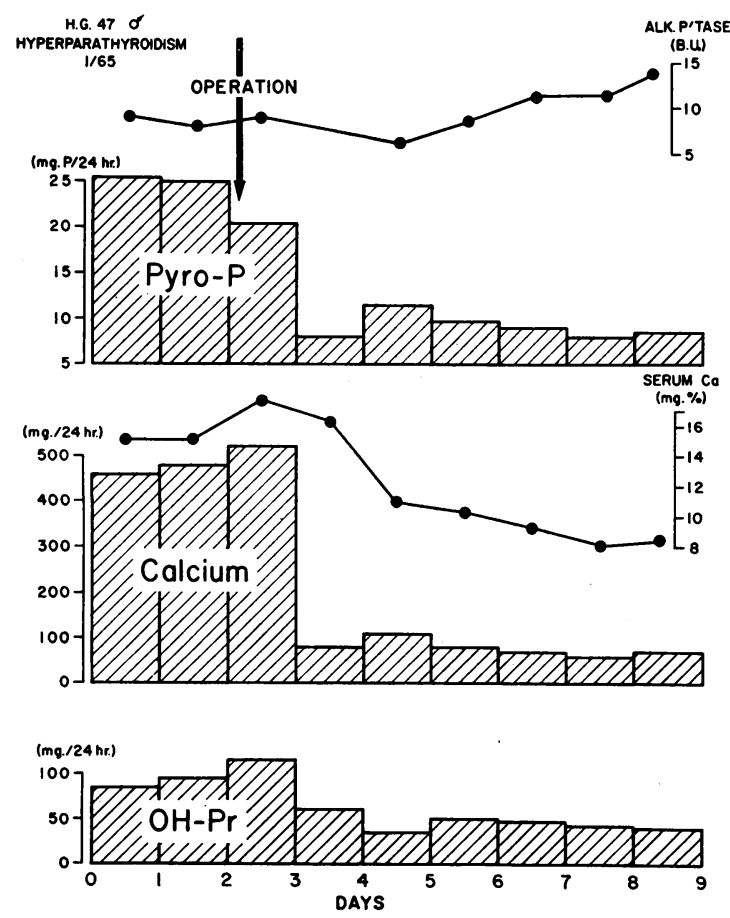

Fig. 1. URINARY PYRophosphate (PYRo-P), HYDROXYPROLINE (OH-PR), AND CALCIUM IN A PATIENT WITH HYPERPARATHYROIDISM BEFORE AND AFTER SURGERY. B.U. = Bodansky units.

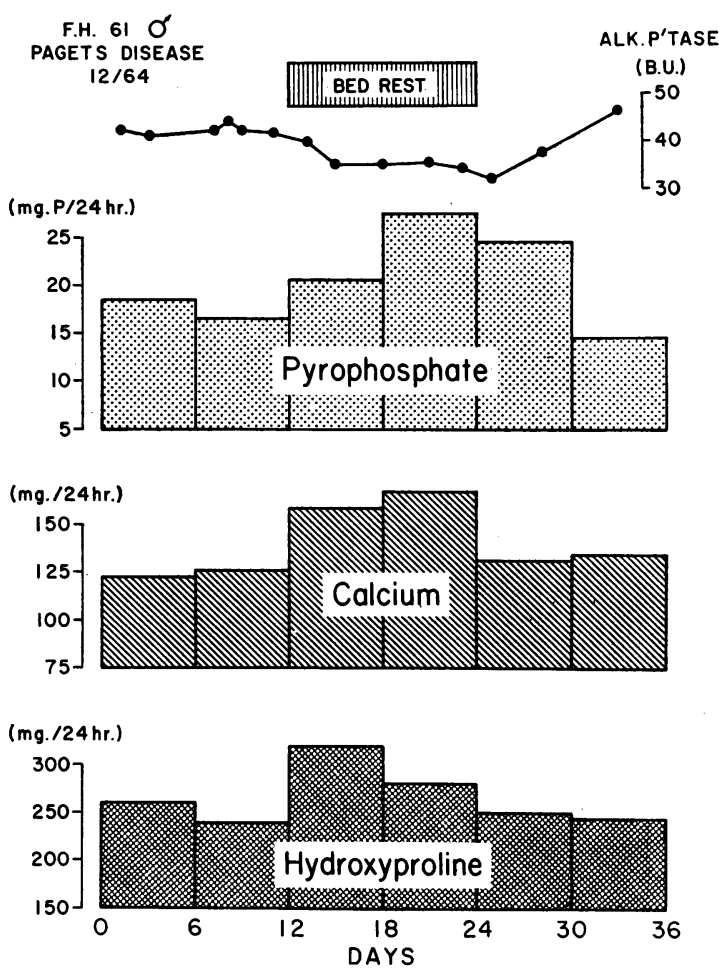

Fig. 2. The efFect of immobilization in Paget's DISEASE ON URINARY HYDROXYPROLINE, CALCIUM, AND PYROPHOSPHATE. The decreased bone formation induced by immobilization was reflected in a fall in serum alkaline phosphatase and concomitant increments in urinary hydroxyproline, calcium, and pyrophosphate. In each instance the vertical bars represent the mean value obtained for 6 consecutive 24-hour urinary determinations. 
drawn slightly and the cannula advanced toward the renal pelvis. The inner needle was then removed and the cannula attached to polyvinyl tubing from a constant speed infusion pump. The total time of renal arterial occlusion was usually less than 60 seconds. A cannula with stylus was also inserted into the carotid artery to facilitate appropriately timed blood sampling. Glomerular filtration rate was estimated by standard clearance techniques. A priming creatinine solution was administered intravenously and immediately followed by a sustaining infusion of creatinine designed to maintain a steady plasma concentration during the experimental period. After a suitable equilibration period, during which glomerular filtration became approximately equal in both kidneys, individual urine collections were begun in graduated cylinders. Both renal arteries were perfused with isotonic saline for three 30 -minute control periods at perfusion rates approximating $1 \mathrm{ml}$ per minute, after which one of the renal arterial perfusates (experimental kidney) was changed to one containing parathyroid extract in isotonic saline. PTE was continuously infused at a rate approximating $1 \mathrm{ml}$ per minute, and observations were continued for a subsequent 3-hour period. Urine was collected separately from each kidney for the measurement of hydroxyproline, pyrophosphate, $\mathrm{Ca}, \mathrm{P}$, and creatinine. Serial blood specimens were drawn midway between each of the timed urine collection periods for measurements of $\mathrm{Ca}, \mathrm{P}$, and creatinine.

Analytic methods. Samples of blood and urine were analyzed for $\mathrm{Ca}$ by flame photometry, for $\mathrm{P}$ by the method of Fiske and Subbarow (5), and for creatinine by the method of Bonsnes and Taussky (6). Samples of urine were also analyzed for inorganic pyrophosphate by a previously published modification of the method of Fleisch, Bisaz, and Care (1) and for total hydroxyproline by the method of Prockop and Udenfriend (7).

\section{Results}

Hyperparathyroidism and Paget's disease. A three- to fourfold reduction in the pyrophosphaturia of subjects with hyperparathyroidism (H.G. and S.F., Table I) was observed in the immediate postoperative period. The fall in pyrophosphate excretion was accompanied by a decrease in serum calcium, and urinary hydroxyproline, orthophosphate, and calcium. As illustrated in Figure 1, these findings coincided with a rise in serum alkaline phosphatase during the postoperative period.

A significant rise in urinary pyrophosphate was observed in both patients with Paget's disease during the period of immobilization (D.F. and F.H., Table I). This was followed by a gradual fall in the ambulatory postcontrol periods toward preimmobilization values. Bed rest also produced significant increments in urinary hydroxyproline and calcium in both patients with little alteration in serum calcium or urinary orthophosphate. In one patient (Figure 2) a fall in serum alkaline phosphatase from 45 to 32 Bodansky $U$ accompanied

TABLE II

Effect of 4-hour calcium infusion on urinary pyrophosphate, orthophosphate, and hydroxyproline*

\begin{tabular}{|c|c|c|c|c|c|c|c|c|c|}
\hline & \multicolumn{3}{|c|}{ Control day } & \multicolumn{3}{|c|}{ Infusion day } & \multicolumn{3}{|c|}{ Postinfusion day } \\
\hline & $\mathbf{P P i}$ & $\mathrm{Pi}$ & $\mathrm{OH}-\mathrm{Pr}$ & $\mathrm{PPi}$ & $\mathbf{P i}$ & $\mathrm{OH}-\mathrm{Pr}$ & $\mathrm{PPi}$ & $\mathbf{P i}$ & $\mathrm{OH}-\mathrm{Pr}$ \\
\hline & $m g P / 24 \mathrm{hr}$ & \multicolumn{2}{|c|}{$m g / 24 h r$} & $\begin{array}{r}m g P / 24 \mathrm{hr} \\
\text { Norm }\end{array}$ & \multicolumn{2}{|c|}{$m g / 24 h r$} & $m g P / 24 \mathrm{hr}$ & \multicolumn{2}{|c|}{$m g / 24 h r$} \\
\hline $\begin{array}{l}\text { K.A. } \\
\text { K.M. } \\
\text { J.B. } \\
\text { I.H. }\end{array}$ & $\begin{array}{l}1.80 \\
4.30 \\
2.61 \\
1.42\end{array}$ & $\begin{array}{l}725 \\
857 \\
515 \\
440\end{array}$ & $\begin{array}{l}28.1 \\
45.7 \\
21.1 \\
28.3\end{array}$ & $\begin{array}{l}0.74 \\
2.10 \\
0.99 \\
0.35\end{array}$ & $\begin{array}{l}333 \\
703 \\
142 \\
253\end{array}$ & $\begin{array}{l}15.7 \\
33.3 \\
12.2 \\
20.5\end{array}$ & $\begin{array}{l}1.49 \\
5.80 \\
0.77 \\
2.82\end{array}$ & $\begin{array}{l}867 \\
984 \\
423 \\
523\end{array}$ & $\begin{array}{l}28.8 \\
38.6 \\
20.9 \\
19.4\end{array}$ \\
\hline $\begin{array}{l}\text { Mean } \\
\text { SE }\end{array}$ & $\begin{array}{r}2.53 \\
\pm \quad 0.97\end{array}$ & $\begin{array}{r}634 \\
\pm 104\end{array}$ & $\begin{array}{r}30: 8 \\
\pm 6.2\end{array}$ & $\begin{array}{r}1.05 \\
\pm 0.44\end{array}$ & $\begin{array}{r}357 \\
\pm 140\end{array}$ & $\begin{array}{r}20.4 \\
\pm 2.1\end{array}$ & $\begin{array}{r}2.72 \\
\pm 1.01\end{array}$ & $\begin{array}{r}699 \\
\pm 140\end{array}$ & $\begin{array}{r}26.9 \\
\pm 4.8\end{array}$ \\
\hline \multicolumn{10}{|c|}{ Hypoparathyroidism } \\
\hline $\begin{array}{l}\text { N.D. } \\
\text { A.D. } \\
\text { A.G. } \\
\text { M.R. }\end{array}$ & $\begin{array}{l}0.84 \\
0.72 \\
1.40 \\
1.12\end{array}$ & $\begin{array}{l}314 \\
627 \\
435 \\
191\end{array}$ & $\begin{array}{r}9.9 \\
13.6 \\
5.5 \\
9.9\end{array}$ & $\begin{array}{l}2.80 \\
1.67 \\
3.40 \\
2.62\end{array}$ & $\begin{array}{l}607 \\
856 \\
556 \\
329\end{array}$ & $\begin{array}{r}13.3 \\
11.0 \\
11.9 \\
8.0\end{array}$ & $\begin{array}{l}3.10 \\
1.49 \\
4.01 \\
1.75\end{array}$ & $\begin{array}{l}653 \\
417 \\
410 \\
135\end{array}$ & $\begin{array}{r}8.2 \\
12.3 \\
9.9 \\
6.4\end{array}$ \\
\hline $\begin{array}{l}\text { Mean } \\
\text { SE }\end{array}$ & $\begin{array}{r}1.02 \\
\pm 0.17\end{array}$ & $\begin{array}{r}392 \\
\pm 109\end{array}$ & $\begin{array}{r}9.7 \\
\pm 2.0\end{array}$ & $\begin{array}{r}2.62 \\
\pm 0.43\end{array}$ & $\begin{array}{r}587 \\
\pm 132\end{array}$ & $\begin{array}{r}11.1 \\
\pm 1.3\end{array}$ & $\begin{array}{r}2.58 \\
\pm 0.63\end{array}$ & $\begin{array}{r}403 \\
\pm 129\end{array}$ & $\begin{array}{r}9.1 \\
\pm 1.5\end{array}$ \\
\hline \multicolumn{10}{|c|}{ Hyperparathyroidism } \\
\hline $\begin{array}{l}\text { W.G. } \\
\text { S.F. }\end{array}$ & $\begin{array}{l}28.6 \\
12.3\end{array}$ & $\begin{array}{r}606 \\
1,330\end{array}$ & $\begin{array}{l}48.6 \\
80.3\end{array}$ & $\begin{array}{r}12.6 \\
9.3\end{array}$ & $\begin{array}{l}574 \\
980\end{array}$ & $\begin{array}{l}36.9 \\
70.6\end{array}$ & $\begin{array}{l}10.1 \\
11.2\end{array}$ & $\begin{array}{r}719 \\
1,032\end{array}$ & $\begin{array}{l}53.7 \\
89.4\end{array}$ \\
\hline
\end{tabular}

* Abbreviations as noted in Table I. 


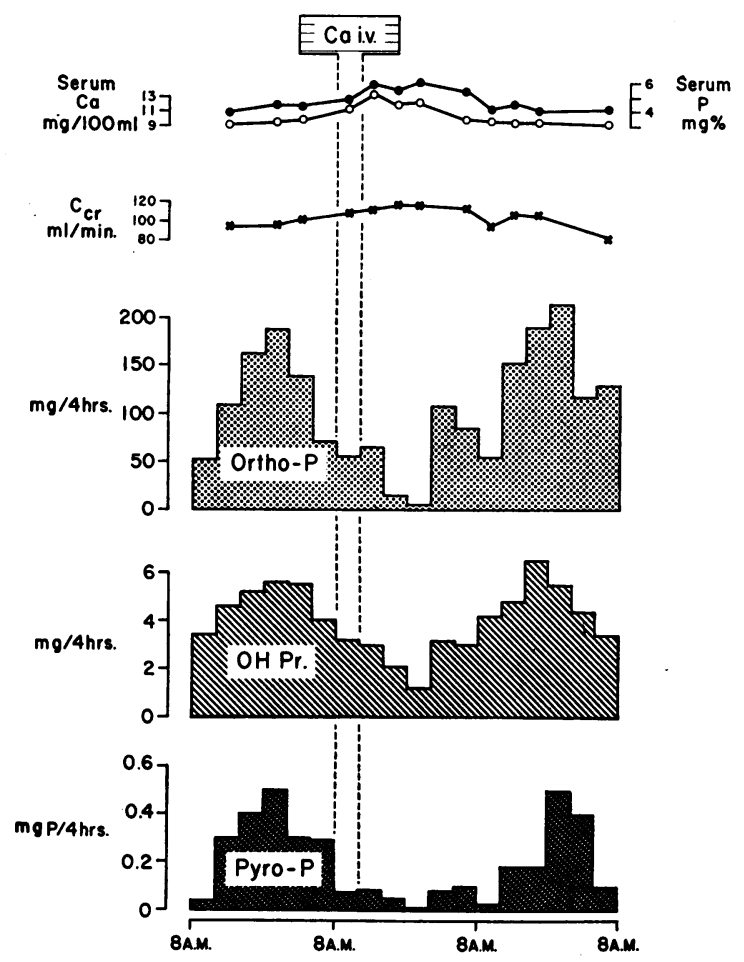

Fig. 3. EFFects of a 4-HOUR CALCIUM infusion on URINARY ORTHOPHOSPHATE (ORTHO-P), HYDROXYPROLINE (OH-Pr), PYROPhOSPhate (PYRO-P), AND CREATININe ClEARANCE $\left(\mathrm{C}_{\mathbf{c r}}\right)$ IN a NORMal wOMan. The closed and open circles refer to serum calcium and phosphorus values, respectively.

bed rest with a rise toward preimmobilization levels in the postcontrol periods.

Calcium infusion. The comparative effects of a sustained 4-hour calcium infusion in normal, hypoparathyroid, and hyperparathyroid subjects are shown in Table II. In the normal subjects, calcium infusion produced a $59 \%$ fall in pyrophosphate excretion from a mean control value of 2.53 $( \pm 0.97)$ to $1.05( \pm 0.44) \mathrm{mg} \mathrm{P}$ per 24 hours. In three of the four control subjects (K.A., K.M., and I.H., Table II), pyrophosphate returned promptly to control values on the first postinfusion day. Decreases in the urinary hydroxyproline and orthophosphate were also observed on the infusion day, averaging 33 and $43 \%$ of control values, respectively. A typical response of a normal subject to the 4-hour calcium infusion is represented graphically in Figure 3. Despite a rise in creatinine clearance and serum phosphate, a fall in pyrophosphate excretion followed the calcium infusion with suppression of the normal diurnal excretion pattern. These changes in pyrophosphate excretion in all four normal subjects were closely correlated in time with corresponding changes in urinary orthophosphate and hydroxyproline.

In contrast to the response obtained in normal controls, calcium infusion produced a $157 \%$ increase in urinary pyrophosphate in hypoparathyroid patients from a mean control value of 1.02 $( \pm 0.17)$ to $2.62( \pm 0.43)$. In these subjects the rise in urinary pyrophosphate persisted during the postinfusion day, averaging $153 \%$ of preinfusion values (Table II). Increments in orthophosphate excretion were also noted on the infusion day, averaging $21 \%$ of the preinfusion values. Urinary hydroxyproline responses to 4-hour calcium infusion varied in hypoparathyroid subjects. During the infusion hydroxyproline excretion rose in two

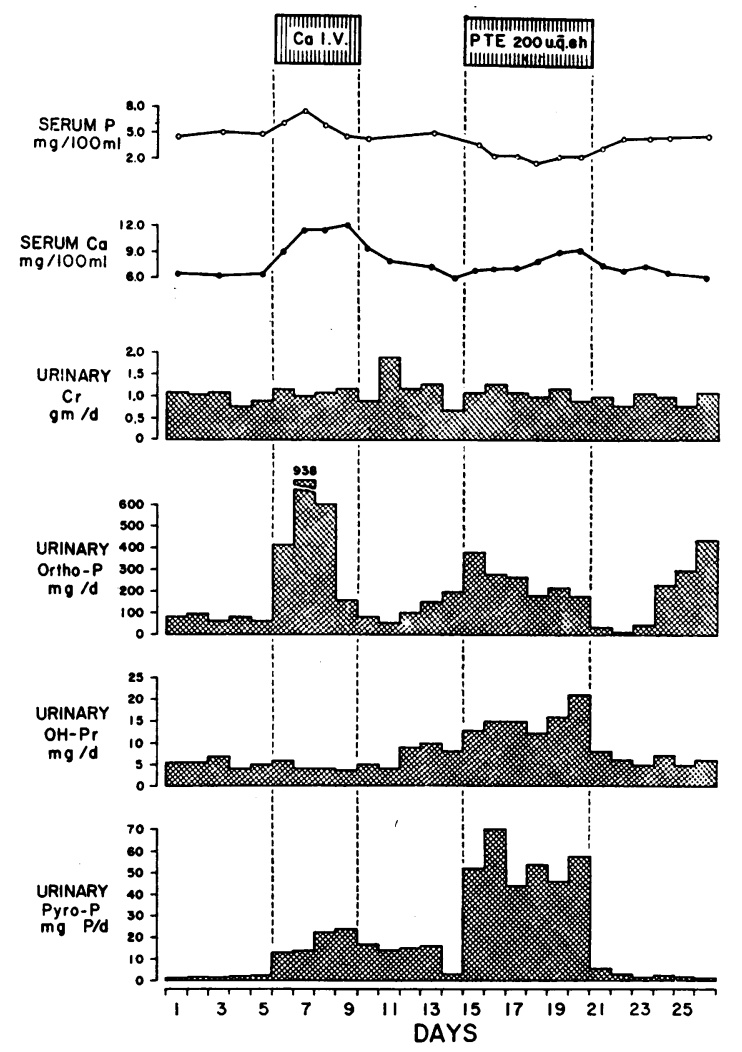

Fig. 4. EFFEct of A 4-DAY CALCIUM INFUSION AND PARATHYROID EXTRACT (PTE) ON SERUM CALCIUM AND PHOSPHORUS, URINARY CREATININE ( $R$ ), ORTHOPHOSPHATE (ORTHO-P), HYDROXYPROLINE (OH-PR), AND PYROPHOSPHATE (PYRO-P) IN A 30-YEAR-OLD FEMALE WITH UNTREATED IDIOPATHIC HYPOPARATHYROIDISM. Two hundred units PTE was administered every 8 hours. 
TABLE III

Effect of parathyroid extract (PTE) on urinary pyrophosphate, orthophosphate, and hydroxyproline*

\begin{tabular}{|c|c|c|c|c|c|c|c|c|c|}
\hline \multirow[b]{2}{*}{ Patient } & \multicolumn{3}{|c|}{ Control } & \multicolumn{3}{|c|}{ PTE } & \multicolumn{3}{|c|}{ Postcontrol } \\
\hline & $\mathbf{P P i}$ & $\mathrm{OH}-\mathrm{Pr}$ & $\mathrm{Pi}$ & $\mathbf{P P i}$ & $\mathrm{OH}-\mathrm{Pr}$ & $\mathrm{Pi}$ & $\mathbf{P P i}$ & $\mathrm{OH}-\mathrm{Pr}$ & $\mathrm{Pi}$ \\
\hline & $m g P / 24 \mathrm{hr}$ & \multicolumn{2}{|c|}{$m g / 24 h r$} & $\begin{array}{r}m g P / 24 h r \\
\text { Norr }\end{array}$ & \multicolumn{2}{|c|}{$m g / 24 h r$} & $m g P / 24 \mathrm{hr}$ & \multicolumn{2}{|c|}{$m g / 24 h r$} \\
\hline $\begin{array}{l}\text { I.H. } \\
\text { I.D. }\end{array}$ & $\begin{array}{l}3.7 \\
2.7\end{array}$ & $\begin{array}{l}19.9 \\
37.4\end{array}$ & $\begin{array}{l}482 \\
596\end{array}$ & $\begin{array}{r}13.7 \\
9.8\end{array}$ & $\begin{array}{l}28.8 \\
59.7\end{array}$ & $\begin{array}{l}569 \\
710\end{array}$ & $\begin{array}{l}3.9 \\
4.1\end{array}$ & $\begin{array}{l}18.1 \\
40.2\end{array}$ & $\begin{array}{l}416 \\
614\end{array}$ \\
\hline \multicolumn{10}{|c|}{ Hypoparathyroidism } \\
\hline $\begin{array}{l}\text { N.D. } \\
\text { A.D. } \\
\text { A.G. } \\
\text { M.R. }\end{array}$ & $\begin{array}{l}1.2 \\
1.5 \\
2.0 \\
1.9\end{array}$ & $\begin{array}{r}13.4 \\
7.1 \\
4.5 \\
18.0\end{array}$ & $\begin{array}{l}680 \\
126 \\
301 \\
352\end{array}$ & $\begin{array}{l}15.2 \\
55.6 \\
12.7 \\
15.3\end{array}$ & $\begin{array}{l}31.3 \\
15.4 \\
15.7 \\
47.6\end{array}$ & $\begin{array}{r}930 \\
454 \\
928 \\
1,005\end{array}$ & $\begin{array}{r}8.9 \\
13.7 \\
3.9 \\
4.8\end{array}$ & $\begin{array}{r}11.2 \\
6.4 \\
5.7 \\
18.1\end{array}$ & $\begin{array}{l}714 \\
181 \\
251 \\
334\end{array}$ \\
\hline $\begin{array}{l}\text { Mean } \\
\text { SE }\end{array}$ & $\begin{array}{r}1.7 \\
\pm 0.2\end{array}$ & $\begin{array}{r}10.7 \\
\pm 3.4\end{array}$ & $\begin{array}{r}365 \\
\pm 138\end{array}$ & $\begin{array}{r}24.7 \\
\pm 10.7\end{array}$ & $\begin{array}{r}27.5 \\
\pm 8.0\end{array}$ & $\begin{array}{r}829 \\
+138\end{array}$ & $\begin{array}{r}7.8 \\
\pm 2.4\end{array}$ & $\begin{array}{r}10.4 \\
\pm 3.1\end{array}$ & $\begin{array}{r}370 \\
\pm 133\end{array}$ \\
\hline
\end{tabular}

* Abbreviations as noted in Table I. Each value in the Table represents the mean of 6 consecutive determinations.

patients (N.D. and A.G., Table II) and fell in two others (A.D. and M.R., Table II).

The results of a prolonged 4-day calcium infusion in one hypoparathyroid subject are illustrated in Figure 4. Coincident with a gradual rise in serum calcium from 6.2 to $12.1 \mathrm{mg}$ per $100 \mathrm{ml}$, urinary pyrophosphate steadily rose from a mean control value of 1.2 to $24.6 \mathrm{mg} P$ per 24 hours on the fourth infusion day. In contrast, increments in urinary orthophosphate were prompt and paralleled corresponding changes in serum inorganic phosphate. Maximal values for both serum phosphate and urinary orthophosphate were reached by the second infusion day followed by a fall in the subsequent 2-day infusion period. No consistent changes were noted in hydroxyproline excretion during this time.

As illustrated in Table II, during a 4-hour calcium infusion in two hypercalcemic subjects with hyperparathyroidism (W.G. and S.F.), a decrease in pyrophosphate excretion approximating 57 and $33 \%$, respectively, of control values was observed. Corresponding decreases in urinary hydroxyproline of 23 and $12 \%$ and in orthophosphate of 5 and $35 \%$ also occurred.

PTE administration. PTE administration for 6 days to two normal subjects (I.H. and I.D., Table III) with mean control pyrophosphate excretion of 3.7 and $2.7 \mathrm{mg} P$ per 24 hours, respectively, resulted in increments to mean values of 13.7 and $9.8 \mathrm{mg} P$ per 24 hours. The results of PTE administration to hypoparathyroid subjects are also summarized in Table III and Figure 4.
The average control urinary pyrophosphate for this group of $1.7 \mathrm{mg} P$ per 24 hours rose 14-fold to 24.7 during treatment with PTE. As illustrated in Figure 4, the PTE-induced pyrophosphaturia was prompt and persisted during the 6-day therapeutic course, returning immediately to pretreatment values the first day PTE was discontinued. During the period of PTE therapy serum phosphate fell to values ranging from 1.9 to $2.5 \mathrm{mg}$ per $100 \mathrm{ml}$, and serum calcium rose to values ranging from 9.7 to $10.9 \mathrm{mg}$ per $100 \mathrm{ml}$. As noted in Table III, when PTE was administered to hypoparathyroid subjects, hydroxyproline and orthophosphate excretion rose to values averaging 156 and $185 \%$ of control.

Renal arterial perfusion. The decrease in pyrophosphate excretion observed while endogenous parathyroid hormone secretion was suppressed in normal subjects by calcium infusions and the pyrophosphaturia after PTE administration to normal and hypoparathyroid subjects suggested a direct relationship between parathyroid activity and pyrophosphate excretion. Since parathyroid hormone exerts a direct influence on renal tubular phosphate transport (4), the observed alterations in pyrophosphate excretion could also have been mediated through PTE-induced changes in the renal excretion of pyrophosphate. For this reason the effect of direct renal arterial perfusion with PTE on pyrophosphate excretion was studied. The protocols of two representative perfusion experiments are shown in Table IV, and a 
TABLE IV

Protocol of two representative renal arterial perfusion experiments*

\begin{tabular}{|c|c|c|c|c|c|c|c|c|c|c|c|}
\hline \multirow{2}{*}{$\begin{array}{l}\text { Dog } \\
\text { no. }\end{array}$} & \multirow[b]{2}{*}{ Time } & \multicolumn{2}{|c|}{$\mathrm{Ccr}$} & \multicolumn{2}{|c|}{ Urine flow } & \multicolumn{2}{|c|}{ UPPiV } & \multicolumn{2}{|c|}{$\mathrm{UP}_{\mathrm{Pi}} \mathrm{V}$} & \multicolumn{2}{|c|}{ UOH - PrV } \\
\hline & & $\overline{\text { Exp. }}$ & Cont. & Exp. & Cont. & Exp. & Cont. & Exp. & Cont. & Exp. & Cont. \\
\hline & $\min$ & \multicolumn{2}{|c|}{$m l / \min$} & \multicolumn{2}{|c|}{$\mathrm{ml} / \mathrm{min}$} & \multicolumn{2}{|c|}{$\mu g / \min$} & \multicolumn{2}{|c|}{$\mu g / \min$} & \multicolumn{2}{|c|}{$\mu g / \min$} \\
\hline & $\begin{array}{r}0-30 \\
30-60 \\
60-90\end{array}$ & $\begin{array}{l}25.4 \\
31.1 \\
27.2\end{array}$ & $\begin{array}{l}26.2 \\
31.9 \\
30.6\end{array}$ & $\begin{array}{l}1.9 \\
1.2 \\
1.2\end{array}$ & $\begin{array}{l}1.3 \\
1.1 \\
1.0\end{array}$ & $\begin{array}{l}3.71 \\
2.74 \\
2.54\end{array}$ & $\begin{array}{l}3.39 \\
4.12 \\
3.58\end{array}$ & $\begin{array}{l}176.1 \\
148.8 \\
190.5\end{array}$ & $\begin{array}{l}148.5 \\
149.3 \\
107.3\end{array}$ & $\begin{array}{l}6.0 \\
7.1 \\
7.5\end{array}$ & $\begin{array}{l}4.7 \\
6.5 \\
6.6\end{array}$ \\
\hline
\end{tabular}

I 90 PTE infusion started in exp. kidney: PTE in isotonic saline at $9.7 \mathrm{U}(0.97 \mathrm{ml})$ per min

$\begin{array}{rrrrrrrrrrr}90-120 & 22.8 & 28.0 & 1.2 & 0.9 & 2.49 & 3.44 & 161.9 & 86.4 & 7.6 & 5.1 \\ 120-150 & 21.8 & 30.3 & 1.2 & 1.0 & 2.03 & 4.41 & 185.0 & 102.2 & 6.7 & 5.7 \\ 150-180 & 21.2 & 28.0 & 1.3 & 1.0 & 2.39 & 3.01 & 206.6 & 120.4 & 6.5 & 5.2 \\ 180-210 & 24.7 & 30.8 & 1.6 & 1.2 & 2.60 & 2.00 & 243.8 & 153.3 & 7.6 & 6.0 \\ 210-240 & 20.2 & 29.3 & 1.9 & 1.3 & 4.03 & 4.25 & 277.9 & 152.0 & 9.0 & 7.0 \\ 0-30 & 24.3 & 23.3 & 2.3 & 1.8 & 3.38 & 4.71 & 195.9 & 191.6 & 10.0 & 10.7 \\ 30-60 & 28.1 & 25.9 & 2.1 & 1.7 & 2.04 & 4.02 & 151.5 & 164.2 & 10.5 & 9.6 \\ 60-90 & 29.0 & 27.0 & 2.0 & 1.8 & 1.32 & 1.07 & 182.5 & 175.6 & 10.3 & 9.8\end{array}$

II 90 PTE infusion started in exp. kidney: PTE in isotonic saline at $10 \mathrm{U}(1.20 \mathrm{ml})$ per min

$\begin{array}{rrrrrrrrrrr}90-120 & 25.6 & 25.7 & 2.2 & 2.3 & 2.10 & 2.80 & 196.5 & 157.5 & 9.6 & 8.5 \\ 120-150 & 28.8 & 25.2 & 2.4 & 2.4 & 1.67 & 2.00 & 216.0 & 180.8 & 10.5 & 8.9 \\ 150-180 & 26.7 & 25.7 & 3.8 & 3.7 & 1.48 & 2.56 & 318.7 & 194.0 & 12.1 & 12.9 \\ 180-210 & 29.5 & 27.2 & 2.7 & 2.8 & 6.37 & 2.13 & 345.1 & 276.5 & 12.3 & 10.1 \\ 210-240 & 28.9 & 27.2 & 1.7 & 2.2 & 8.64 & 9.31 & 354.7 & 309.8 & 10.0 & 13.9\end{array}$

* Abbreviations: exp. = PTE-perfused kidney; cont. = control saline-perfused kidney; $\mathrm{C}_{\mathrm{Cr}}=$ clearance of creatinine; $\mathrm{U}_{\mathrm{PPi}} \mathrm{V}=$ excreted pyrophosphate; $\mathrm{U}_{\mathrm{Pi}} \mathrm{V}=$ excreted orthophosphate; $\mathrm{U}_{\mathrm{OH}-\mathrm{Pr}} \mathrm{V}=$ excreted hydroxyproline.

summary of four such perfusion experiments is rise (within the first 30-minute collection period) noted in Table $\mathrm{V}$.

When unilateral PTE-renal arterial perfusion was initiated in each of four dogs, an immediate in orthophosphate excretion was observed in the perfused kidney in three, with gradual increments during the remainder of the perfusion period ( $\mathrm{Ta}$ -

TABLE $V$

Summary of animal renal arterial perfusion experiments*

\begin{tabular}{|c|c|c|c|c|c|c|c|c|c|c|}
\hline \multirow{2}{*}{$\begin{array}{l}\text { Dog } \\
\text { no. }\end{array}$} & \multirow[b]{2}{*}{ Collection periods } & \multirow[b]{2}{*}{ Infusion } & \multicolumn{2}{|c|}{$\mathrm{C}_{\mathrm{Cr}}$} & \multicolumn{2}{|c|}{$\mathrm{UPP}_{\mathrm{Pi}} \mathrm{V}$} & \multicolumn{2}{|c|}{$\mathrm{U}_{P_{\mathrm{i}} \mathrm{V}}$} & \multicolumn{2}{|c|}{ UоH_Pr $\mathrm{V}$} \\
\hline & & & Exp. & Cont. & Exp. & Cont. & Exp. & Cont. & Exp. & Cont. \\
\hline & $\min$ & $U / \min$ & \multicolumn{2}{|c|}{$m l / m i n$} & \multicolumn{2}{|c|}{$\mu g / \min$} & \multicolumn{2}{|c|}{$\mu g / \min$} & \multicolumn{2}{|c|}{$\mu g / \min$} \\
\hline I & $\begin{array}{l}\text { Control }(0-90) \\
\text { PTE }(90-180) \\
\text { PTE }(180-240)\end{array}$ & $\begin{array}{l}0 \\
9.7 \\
9.7\end{array}$ & $\begin{array}{l}27.9 \\
21.9 \\
22.5\end{array}$ & $\begin{array}{l}29.3 \\
28.7 \\
30.0\end{array}$ & $\begin{array}{l}2.99 \\
2.30 \\
3.31\end{array}$ & $\begin{array}{l}3.69 \\
3.62 \\
3.12\end{array}$ & $\begin{array}{l}171.8 \\
184.5 \\
260.8\end{array}$ & $\begin{array}{l}135.0 \\
103.0 \\
152.6\end{array}$ & $\begin{array}{l}6.88 \\
6.98 \\
8.34\end{array}$ & $\begin{array}{l}5.96 \\
5.36 \\
6.50\end{array}$ \\
\hline II & $\begin{array}{l}\text { Control }(0-90) \\
\text { PTE }(90-180) \\
\text { PTE }(180-240)\end{array}$ & $\begin{array}{r}0 \\
10 \\
10\end{array}$ & $\begin{array}{l}27.1 \\
27.0 \\
29.2\end{array}$ & $\begin{array}{l}28.7 \\
25.5 \\
27.2\end{array}$ & $\begin{array}{l}2.24 \\
1.75 \\
7.50\end{array}$ & $\begin{array}{l}3.26 \\
2.45 \\
5.72\end{array}$ & $\begin{array}{l}176.6 \\
243.7 \\
349.9\end{array}$ & $\begin{array}{l}177.1 \\
167.4 \\
293.2\end{array}$ & $\begin{array}{l}10.26 \\
10.73 \\
11.15\end{array}$ & $\begin{array}{l}10.01 \\
10.10 \\
12.00\end{array}$ \\
\hline III & $\begin{array}{l}\text { Control }(0-90) \\
\text { PTE }(90-180) \\
\text { PTE }(180-240)\end{array}$ & $\begin{array}{r}0 \\
12 \\
12\end{array}$ & $\begin{array}{l}26.8 \\
25.3 \\
27.1\end{array}$ & $\begin{array}{l}27.4 \\
24.3 \\
25.6\end{array}$ & $\begin{array}{l}1.97 \\
2.03 \\
4.04\end{array}$ & $\begin{array}{l}2.61 \\
2.14 \\
3.98\end{array}$ & $\begin{array}{l}186.3 \\
347.4 \\
412.3\end{array}$ & $\begin{array}{l}164.2 \\
171.3 \\
201.5\end{array}$ & $\begin{array}{r}9.73 \\
9.24 \\
11.32\end{array}$ & $\begin{array}{r}8.79 \\
9.01 \\
12.00\end{array}$ \\
\hline IV & $\begin{array}{l}\text { Control }(0-90) \\
\text { PTE }(90-180) \\
\text { PTE }(180-240)\end{array}$ & $\begin{array}{l}0 \\
10.5 \\
10.5\end{array}$ & $\begin{array}{l}29.7 \\
31.5 \\
30.2\end{array}$ & $\begin{array}{l}30.1 \\
29.9 \\
31.4\end{array}$ & $\begin{array}{l}3.42 \\
3.74 \\
4.89\end{array}$ & $\begin{array}{l}2.71 \\
2.03 \\
5.14\end{array}$ & $\begin{array}{l}201.4 \\
467.3 \\
499.2\end{array}$ & $\begin{array}{l}198.3 \\
204.6 \\
373.7\end{array}$ & $\begin{array}{l}6.51 \\
5.97 \\
8.37\end{array}$ & $\begin{array}{r}7.33 \\
8.10 \\
10.26\end{array}$ \\
\hline $\begin{array}{l}\text { Mean } \\
\text { values } \dagger\end{array}$ & $\begin{array}{l}\text { Control }(0-90) \\
\text { PTE }(90-180) \\
\text { PTE }(180-240)\end{array}$ & & $\begin{array}{l}27.9 \\
26.4 \\
27.3\end{array}$ & $\begin{array}{l}28.8 \\
27.1 \\
28.5\end{array}$ & $\begin{array}{l}2.66 \\
2.45 \\
4.64\end{array}$ & $\begin{array}{l}3.06 \\
2.78 \\
3.71\end{array}$ & $\begin{array}{l}184.0 \\
310.7 \\
380.6\end{array}$ & $\begin{array}{l}168.6 \\
161.6 \\
255.3\end{array}$ & $\begin{array}{l}8.35 \\
8.23 \\
9.79\end{array}$ & $\begin{array}{r}8.02 \\
8.14 \\
10.19\end{array}$ \\
\hline
\end{tabular}

* Abbreviations as in Table IV. Rate of PTE infusion into renal artery. Each value in this Table is the mean of two or three 30-minute clearance periods.

$\dagger$ Mean values for both experimental and control kidneys before and during PTE infusion in dogs I, II, III, IV. 
bles IV and V and Figure 5). In contrast, significant increments in pyrophosphate excretion by the experimental kidney were delayed for $1 \frac{1}{2}$ to 2 hours after the PTE infusion had begun and coincided with similar delayed increments in pyrophosphate excretion from the control saline-perfused kidney (Figure 5). As noted in Table V, the control kidney shared in this delayed response to PTE with increments in orthophosphate, pyrophosphate, and hydroxyproline excretion. These characteristic delayed increments in pyrophosphate excretion by both PTE- and saline-perfused kidneys occurred in the absence of any significant differential changes in creatinine clearance or urine flow.

\section{Discussion}

Pyrophosphate, an anhydro-dimer of orthophosphate present in most body tissues, plays an integral role in cellular biosynthesis $(8,9)$. It has been identified in bone by Cartier and Picard (10) and Perkins and Walker (11) and its possible role in hydroxyapatite precipitation and crystal growth reviewed by Fleisch and Bisaz (12, 13). Recently Krane and Glimcher have also cited evidence that inorganic pyrophosphate is bound to the calcium apatite crystal (14). Despite these observations the significance of urinary pyrophosphate and its relation to bone metabolism are still uncertain. The pyrophosphaturia previously reported in hyperparathyroidism and Paget's disease (1) suggested that pyrophosphate excretion reflected the accelerated bone resorption characteristic of these disorders. The present findings are consistent with the hypothesis that pyrophosphate excretion is also controlled to some extent by parathyroid activity. A prompt pyrophosphaturia was observed in normal and hypoparathyroid subjects during PTE administration, and conversely, a significant fall in pyrophosphate excretion occurred only in normal subjects during suppression of endogenous parathyroid hormone release by calcium infusion. That this parathyroid control is not mediated through renal phosphate regulating mechanisms is suggested by the renal arterial perfusion experiments in dogs wherein no direct effect of PTE on pyrophosphate excretion was observed (Tables IV and V, Figure 5).

The delayed bilateral rise in pyrophosphate excretion observed during unilateral renal arterial

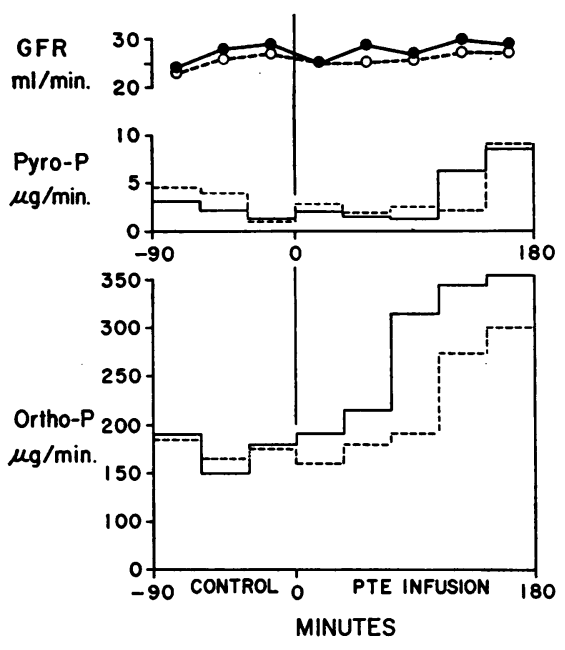

Fig. 5. EFFEctS OF INTRARENAL INFUSION OF PARATHYROID EXTRACT (PTE). The vertical line at zero time indicates the start of the parathyroid extract infusion in the experimental kidney. Urinary orthophosphate (ortho-P) and pyrophosphate (pyro-P) excretion rates are illustrated on the left. Simultaneously obtained values for glomerular filtration rate (GFR) are shown at the top of the Figure. The solid lines represent the experimental (parathyroid extract-infused) and the dotted lines the control (isotonic saline-infused) kidney.

perfusion with PTE is consistent with a systemic effect of nonfiltered PTE. Hydroxyprolinuria has often been reported in clinical disorders of bone metabolism $(1,15-17)$, and an apparent relation between parathyroid activity and urinary hydroxyproline has been defined (17). Recent isotopic data also reveal that hydroxyproline excretion correlates well with bone resorption (1820 ). The delayed hydroxyprolinuria observed in both kidneys during unilateral renal arterial perfusion with PTE probably reflects bone resorption induced by the nonfiltered fraction of infused PTE. In view of the PTE-pyrophosphate relationship observed in man (Tables II and III), the delayed pyrophosphaturia noted in the dog perfusion experiments may also be related to PTEinduced bone resorption.

The results of postsurgical studies in hyperparathyroidism and immobilization in Paget's disease suggest that pyrophosphate like hydroxyproline excretion derives primarily from bone resorption. In both patients with hyperparathyroidism, a prompt reduction in urinary pyrophosphate occurred postoperatively when bone destruction was presumably decreased and reparative bone forma- 
tion proceeding at a rapid rate as indicated by a rising serum alkaline phosphatase (Figure 1). Hypercalciuria, hydroxyprolinuria, and a fall in serum alkaline phosphatase have been observed when patients with Paget's disease are immobilized and attributed to a combination of decreased bone formation and increased bone resorption (21, 22). In the present study, immobilization produced a rise in urinary pyrophosphate in two subjects with active Paget's disease as well as hydroxyprolinuria and hypercalciuria (Table I). As illustrated in Figure 2, the increase in pyrophosphate excretion occurred despite a fall in bone formation rate as reflected by steadily decreasing serum alkaline phosphatase.

Comparable diurnal excretory patterns of orthoand pyrophosphate illustrated in Figure 3 and the parallel rise and fall of both ortho- and pyrophosphate during calcium infusion studies in hypoparathyroid and normal subjects, respectively, suggest that pyrophosphate excretion may also be regulated by orthophosphate excretion. Proportional increments in ortho- and pyrophosphate excretion have been observed in man during orthophosphate feeding experiments (23-25). Russell, Edwards, and Hodgkinson have proposed several possible mechanisms for an orthophosphate control of pyrophosphate excretion, including repressions of inorganic pyrophosphatases by orthophosphate and competition between ortho- and pyrophosphate for renal tubular resorption (25). It could also be postulated that elevations in urinary pyrophosphate relate directly to increments in the endogenous phosphate pool resulting in an accelerated formation of pyrophosphate. However, not only are the thermodynamic requirements for a direct endergonic orthophosphatepyrophosphate transformation impractical at physiological $\mathrm{pH}$ (26), but in the presence of the widely distributed enzyme, inorganic pyrophosphatase, the pyrophosphate would disappear almost as promptly as it was formed (8). The hypothesis that the changes in urinary pyrophosphate, illustrated for normal subjects in Table I, resulted primarily from suppression of endogenous parathyroid hormone release and decreased bone resorption rather than from secondary changes in circulating phosphate and orthophosphate excretion is strengthened by the observation that pyrophosphate excretion falls when calcium salts are administered to normal individuals despite a concomitant rise in serum phosphate (Figure 3). Whereas the coincident fall in orthophosphate clearance under these circumstances is attributed to an increased tubular resorption of orthophosphate $(27-29)$, the fall in urinary pyrophosphate probably reflects a decrease in bone resorption, since PTE has no direct effect on the renal excretion of pyrophosphate (Tables IV and V, Figure 5).

The source of the pyrophosphate that brings about the rise in urinary levels in patients with hypoparathyroidism during calcium infusion can only be speculated upon at this time. Short term infusion of calcium salts to these patients generally produces a rise in both serum phosphate and orthophosphate clearance $(29,30)$. The rise in orthophosphate excretion has been attributed in the past to the rise in serum phosphorus coincident with a decreased tubular resorption of phosphate. Recently Eisenberg has shown that the increments in orthophosphate clearance in hypoparathyroid subjects during calcium infusion are also mediated through a mechanism for phosphate homeostasis that is independent of parathyroid control (31). As noted in Figure 4, the increments in pyrophosphate excretion observed during a prolonged 4-day infusion in a subject with hypoparathyroidism coincided with increments in serum calcium levels rather than with changes in serum phosphate. It seems unlikely that the skeleton would yield pyrophosphate in response to hypercalcemia, since hypercalcemia should cause deposition of bone rather than resorption. The most reasonable explanation for the pyrophosphaturia observed in hypoparathyroid subjects during a period of induced hypercalcemia is that the hypercalcemia per se causes an increase in cellular pyrophosphate (possibly via an inhibition of the enzyme, inorganic pyrophosphatase) and a shift of cellular pyrophosphate to the extracellular compartment. A consequent increase in circulating pyrophosphate would then result in pyrophosphaturia. The estimated low pyrophosphate blood concentration of $10^{-5}$ mole per L (13) and lack of specificity in available quantitative methods for serum pyrophosphate presently prohibit the serum pyrophosphate measurements essential for verification of this hypothesis. 
A fall in urinary pyrophosphate, orthophosphate, and hydroxyproline was observed in two patients with hyperparathyroidism during short term infusion with calcium salts (Table II). Schwartz (32) and Nordin, Smith, and MacGregor (33) have also noted suppression of hydroxyproline excretion in subjects with hyperparathyroidism during calcium infusion studies despite the reported insensitivity of parathyroid tumors to induced hypercalcemia $(27,29)$. These results suggest either that some parathyroid tumors may not be completely autonomous and their secretions alterable by physiologic stimuli or that in weakly functioning tumors the remaining normal glands may be still further suppressed by induced hypercalcemia.

\section{Summary}

Parathyroid extract administration to normal subjects and patients with hypoparathyroidism caused a significant increase in urinary pyrophosphate that reverted to pretreatment values when therapy was discontinued. A 4-hour constant infusion of calcium produced a prompt fall in pyrophosphate excretion in normal subjects but not in hypoparathyroid patients.

Unilateral renal arterial perfusion with parathyroid extract in dogs resulted in a significant prompt rise in orthophosphate and a delayed rise in pyrophosphate excretion. The latter coincided with corresponding delayed increments in orthophosphate, hydroxyproline, and pyrophosphate excretion in the contralateral control saline-perfused kidney. These observations suggest that pyrophosphate excretion is regulated by parathyroid activity and that this regulation is mediated through alterations in bone resorption.

\section{Acknowledgments}

The authors gratefully acknowledge the assistance of Misses Janeann Prout and Maureen Burke. We are also grateful to Miss Olga Kennedy, dietitian, and Mr. Peter Ignatenko, medical illustrator, of the Clinical Research Center.

\section{References}

1. Avioli, L. V., J. E. McDonald, and R. A. Singer. Excretion of pyrophosphate in disorders of bone metabolism. J. clin. Endocr. 1965, 25, 912.

2. McLean, F. C., and W. Bloom. Calcification and ossification. Mobilization of bone salt by parathyroid extract. Arch. Path. 1941, 32, 315.
3. Chang, H.-Y. Grafts of parathyroid and other tissues to bone. Anat. Rec. 1951, 111, 23.

4. Pullman, T. N., A. R. Lavender, I. Aho, and H. Rasmussen. Direct renal action of a purified parathyroid extract. Endocrinology 1960, 67, 570.

5. Fiske, C. H., and Y. Subbarow. The colorimetric determination of phosphorus. J. biol. Chem. 1925, 66, 375.

6. Bonsnes, R. W., and H. H. Taussky. On the colorimetric determination of creatinine by the Jaffe reaction. J. biol. Chem. 1945, 158, 581.

7. Prockop, D. J., and S. Udenfriend. A specific method for the analysis of hydroxyproline in tissues and urine. Analyt. Biochem. 1960, 1, 228.

8. Stetten, D., Jr. Biosynthesis and pyrophosphate. Amer. J. Med. 1960, 28, 867.

9. Stetten, M. R. Metabolism of inorganic pyrophosphate. III. Acceptor specificity studies with rat liver microsomal pyrophosphate phosphotransferase activity. J. biol. Chem. 1965, 240, 2248.

10. Cartier, P., and J. Picard. La minéralisation du cartilage ossifiable: I. La mineralisation du cartilage in vitro. Bull. Soc. Chim. biol. (Paris) $1955,37,485$.

11. Perkins, H. R., and P. G. Walker. The occurrence of pyrophosphate in bone. J. Bone Jt Surg. 1958, 40, 333.

12. Fleisch, H., and S. Bisaz. Isolation from urine of pyrophosphate, a calcification inhibitor. Amer. J. Physiol. 1962, 203, 671.

13. Fleisch, H., and S. Bisaz. Mechanism of calcification: inhibitory role of pyrophosphate. Nature (Lond.) 1962, 195, 911.

14. Krane, S. M., and M. J. Glimcher. Transphosphorylation from nucleoside di- and triphosphates by apatite crystals. J. biol. Chem. 1962, 237, 2991.

15. Benoit, F. L., G. B. Theil, and R. H. Watten. Hydroxyproline excretion in endocrine disease. Metabolism 1963, 12, 1072.

16. Dull, T. A., and P. H. Henneman. Urinary hydroxyproline as an index of collagen turnover in bone. New Engl. J. Med. 1963, 268, 132.

17. Keiser, H. R., J. R. Gill, Jr., A Sjoerdsma, and F. C. Bartter. Relation between urinary hydroxyproline and parathyroid function. J. clin. Invest. 1964, 43, 1073.

18. Avioli, L. V., and D. J. Prockop. Collagen metabolism and the response to parathyroid extract in the intact rhesus monkey. Clin. Res. 1965, 13, 545.

19. Prockop, D. J. Isotopic studies on collagen degradation and the urine excretion of hydroxyproline. J. clin. Invest. 1964, 43, 453.

20. Klein, L., F. W. Lafferty, O. H. Pearson, and P. H. Curtiss, Jr. Correlation of urinary hydroxyproline, serum alkaline phosphatase and skeletal calcium turnover. Metabolism 1964, 13, 272.

21. The Parathyroid Glands and Metabolic Bone Disease, F. Albright and E. C. Reifenstein, Eds. Baltimore, Williams \& Wilkins, 1948, pp. 290-299. 
22. Henneman, P. H., T. A. Dull, L. V. Avioli, C. H. Bastomsky, and T. N. Lynch. Effect of aspirin and corticosteroids on Paget's disease of bone. Trans. Coll. Phycns Philad. 1963, 31, 10.

23. Fleisch, H., S. Bisaz, and A. D. Care. Effect of orthophosphate on urinary pyrophosphate excretion and the prevention of urolithiasis. Lancet 1964, 1, 1065.

24. McIntosh, H. W., and B. M. Carruthers. Urinary excretion of pyrophosphate in recurrent calcium stone formers. Clin. Res. 1965, 13, 136.

25. Russell, R. G. G., N. A. Edwards, and A. Hodgkinson. Urinary pyrophosphate and urolithiasis. Lancet 1964, 1, 1446.

26. George, P., and R. J. Rutman. The "high energy phosphate bond concept in Progress in Biophysics and Biophysical Chemistry, J. A. V. Butler and B. Katz, Eds. New York, Pergamon, 1960, p. 20.

27. Kyle, L. H., M. Schaaf, and L. A. Erdman. The metabolic effects of intravenous administration of calcium. J. Lab. clin. Med. 1954, 43, 123.

28. Hiatt, H. H., and D. D. Thompson. Some effects of intravenously administered calcium on inorganic phosphate metabolism. J. clin. Invest. 1957, 36, 573.

29. Howard, J. E., T. R. Hopkins, and T. B. Connor. On certain physiologic responses to intravenous injection of calcium salts into normal, hyperparathyroid and hypoparathyroid persons. J. clin. Endocr. 1953, 13, 1.

30. Goldman, R., and S. H. Bassett. Effect of intravenous calcium gluconate upon the excretion of calcium and phosphorus in patients with idiopathic hypoparathyroidism. J. clin. Endocr. 1954, 14, 278.

31. Eisenberg, E. Effects of serum calcium level and parathyroid extracts on phosphate and calcium excretion in hypoparathyroid patients. J. clin. Invest. $1965,44,942$.

32. Schwartz, T. B. Editorial comment in Year Book of Endocrinology. Chicago, Year Book, 1964-65, p. 119.

33. Nordin, B. E. C., D. A. Smith, and J. MacGregor. Parathyroid hormone and blood-bone equilibrium in The Parathyroid Glands, P. J. Gaillard, R. V. Talmage, and A. M. Budy, Eds. Chicago, University of Chicago Press, 1965, p. 125.

\section{SPECIAL NOTICE TO SUBSCRIBERS}

Post Offices will no longer forward the Journal when you move.

Please notify The Journal of Clinical Investigation, Business

Office, 10 Stoughton Street, Boston, Mass. 02118, at once when you have a change of address, and do not omit the $\mathrm{Zip}$ Code number. 